\title{
Low-Level Laser Therapy and Calcitonin in Bone Repair: Densitometric Analysis
}

\author{
Tatiana Pinto Ribeiro, ${ }^{1}$ Simone Bustamante Nascimento, ${ }^{1}$ Claúdia Alessandra Cardoso, ${ }^{1}$ \\ Raduan Hage, ${ }^{2}$ Janete Dias Almeida, ${ }^{3}$ and Emilia Angela Loschiavo Arisawa ${ }^{4}$ \\ ${ }^{1}$ Universidade do Vale do Paraíba (UNIVAP), 12244-000 São José dos Campos, SP, Brazil \\ ${ }^{2}$ Health Science Faculty, Universidade do Vale do Paraíba (UNIVAP), 12244-000 São José dos Campos, SP, Brazil \\ ${ }^{3}$ Department of Biosciences and Oral Diagnosis, São José dos Campos Dental School, São Paulo State University (UNESP), \\ 12245-000, São José dos Campos, SP, Brazil \\ ${ }^{4}$ Biomedical Vibrational Spectroscopy Laboratory, Research and Development Institute (IP\&D), \\ Universidade do Vale do Paraíba (UNIVAP), 12244-000 São José dos Campos, SP, Brazil
}

Correspondence should be addressed to Emilia Angela Loschiavo Arisawa, mirela@univap.br

Received 24 January 2012; Revised 5 April 2012; Accepted 6 April 2012

Academic Editor: Jincai Zhao

Copyright ( $) 2012$ Tatiana Pinto Ribeiro et al. This is an open access article distributed under the Creative Commons Attribution License, which permits unrestricted use, distribution, and reproduction in any medium, provided the original work is properly cited.

\begin{abstract}
The aim of this work was to evaluate the association of low-level laser therapy (LLLT, $830 \mathrm{~nm}$ ) and calcitonin in bone repair considering that bone healing remains a challenge to health professionals. Calcitonin has antiosteoclastic action and LLLT is a treatment that uses low-level lasers or light-emitting diodes to alter cellular function. Both are used to improve bone healing. Densitometry is a clinical noninvasive valuable tool used to evaluate bone mineral density (BMD). Sixty male rats were submitted to bone defect with a trephine bur, randomly divided into four groups of 15 animals each: control (C); synthetic salmon calcitonin (Ca); LLLT (La); LLLT combined with calcitonin ( $\mathrm{LaCa}$ ). Animals from $\mathrm{Ca}$ and $\mathrm{LaCa}$ received $2 \mathrm{UI} / \mathrm{Kg}$ synthetic salmon calcitonin intramuscularly on alternate days after surgery. Animals from groups La and LaCa were treated with infrared LLLT (830 $\mathrm{nm}$, $10 \mathrm{~mW}, 20 \mathrm{~J} / \mathrm{cm}^{2}, 6 \mathrm{~s}$, contact mode). Five animals from each group were euthanized 7, 14, and 21 days after surgery and bone defects were analyzed by densitometry. Statistical analysis showed a significant difference in BMD values in LaCa group at 7 and 21 days $(P=0,005)$. The results of the densitometric study showed that LLLT $(830 \mathrm{~nm})$ combined with calcitonin improved bone repair.
\end{abstract}

\section{Introduction}

Bone healing of large defects is still a challenge to health professionals [1-3]. During the remodeling process, several cytokines, peptides, and growth factors are released locally. Bone-formation markers include serum osteocalcin, bonespecific alkaline phosphatase, and procollagen I carboxyterminal propeptide [4].

Calcitonin has analgesic, anti-inflammatory, and antiosteoclastic actions, therefore, it is used to treat clinical and biological diseases characterized by excessive human bone remodeling $[1,2,5]$. The treatment with calcitonin helps to accelerate healing of bone defects in rats $[2,5]$. Calcitonin action becomes more evident during repair in the initial phases of osteogenesis by stimulating bone formation [2, $3,5]$. Salmon calcitonin cannot only increase bone mineral density (BMD) in osteoporotic bone but also enhance the bone biomechanical properties and improve the healing process in fractured osteoporotic bone [6].

Different techniques have been used in dentistry with an ultimate aim of improving the bone quality. Low-level laser therapy (LLLT) has been used to improve bone healing in several conditions such as in alveolus of dental extraction, bone fractures, [7] dental implants, [8-10] orthodontic treatment, and orthognatic surgery [11].

Several studies have demonstrated that the nonsurgical near-infrared laser is more suitable for bone repair, due to deeper penetration in bone tissue when compared to 


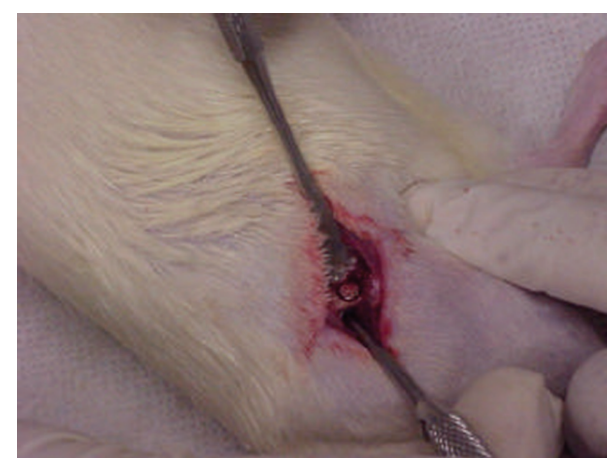

(a)

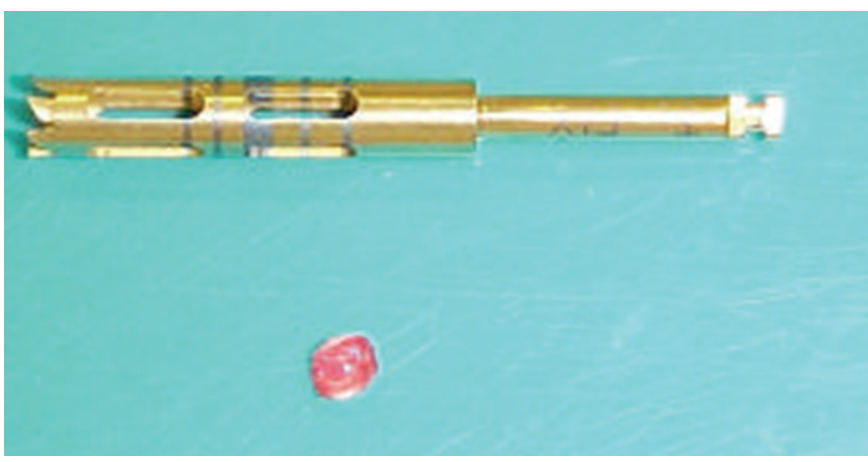

(b)

FIGURE 1

TABLe 1: Parameters of laser protocol.

\begin{tabular}{lccccc}
\hline Groups & Wavelenght & Energy density & Potency & Time irradiated & Area $\left(\mathrm{cm}^{2}\right)$ \\
\hline La and $\mathrm{LaCa}$ & $830 \mathrm{~nm}$ & $20 \mathrm{~J} / \mathrm{cm}^{2}$ & $10 \times 10^{-3} \mathrm{~W}$ & $6 \mathrm{~s}$ & 0.002827 \\
\hline
\end{tabular}

visible laser [12]. Although the use of LLLT on bone healing biomodulation has been growing steadily and several studies have demonstrated positive results on the healing of bone tissue, there are no reports on the combination of LLLT and calcitonin [3].

Prado-Filho and Sterman [13] used radiographic optical densitometry (ROD) in the evaluation of mineral bone density in thoroughbred horses in an initial training program. ROD is a quantitative analysis used to quantify bone mineral density (BMD) in equivalence to aluminum millimeters (mmAL) $[2,3,9]$.

In this study, ROD was used to evaluate BMD after LLLT $(830 \mathrm{~nm})$ combined with calcitonin to measure the improvement in bone repair in surgical defects in rats.

\section{Materials and Methods}

Sixty male Wistar rats, weighting an average of $250 \mathrm{~g}$, were used in this study and were maintained in accordance to the guidelines of the committee on care and use of laboratory animals of the Brazilian National Research Council and the Research Ethic Committee at the Universidade do Vale do Paraíba, Brazil (L036/2003/CEP). The rats had water and food available ad libitum. The animals were randomly distributed into four groups of 15 animals each, control group (C); synthetic salmon calcitonin (Ca); LLLT (La); LLLT combined with calcitonin ( $\mathrm{LaCa}$ ). These groups were subdivided into three subgroups of 5 animals according to the time of euthanasia.

Surgical bone defects in the right femur were made under general anesthetic, using Acepromazine $1 \mathrm{mg} / \mathrm{kg}$ (Acepran $0,2 \%$, Univet S.A), Butorfanol $1 \mathrm{mg} / \mathrm{kg}$ (Lab. Strong Dodge Ltda) and Zolazepan/Tiletamine $15 \mathrm{mg} / \mathrm{Kg}$ (Zoletil, Lab.Virbac S.A). A longitudinal incision on the skin and subcutaneous tissue was made. After femur exposure, a mechanical bone defect was created by a low-speed drill with a $2.8 \mathrm{~mm}$ trephine bur (Figure 1) kept under constant

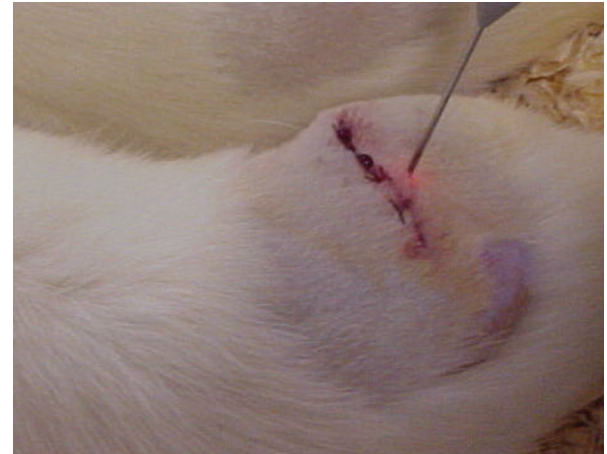

Figure 2 refrigeration with a sterile $0.9 \%$ saline solution. The muscle and subcutaneous tissue was sutured using catgut 4.0 (Cirumédica, Cotia, SP, Brazil), and the skin was sutured with silk 4.0 (Ethicon, Johnson \& Johnson, São José dos Campos, SP, Brazil).

The animals from the $\mathrm{Ca}$ and $\mathrm{LaCa}$ groups received $2 \mathrm{UI} / \mathrm{Kg}$ synthetic salmon calcitonin (Miacalcic, Novartis SA) intramuscularly immediately after the surgical procedure and then on alternate days until euthanasia. Groups La and LaCa were irradiated in a contact mode with a continuous wave (CW) $830 \mathrm{~nm}$ diode laser, with mean optical output power of $10 \mathrm{~mW}$, energy density of $20 \mathrm{~J} / \mathrm{cm}^{2}$, time irradiated $3 \mathrm{~min} 33 \mathrm{~s}$, and area $0.1 \mathrm{~cm}^{2}$ (model: thera Laser, D. M. C. Equipamentos Ltda., São Carlos, SP, Brazil). The parameters of laser protocol used in this study are presented in Table 1. The LLLT was applied transcutaneously, with the hand piece perpendicularly positioned to the wound (Figure 2). The $830 \mathrm{~nm}$ laser showed a $0.4 \mathrm{~nm}$ wavelength drift from the cold to warm operation conditions. Stabilization at $834 \mathrm{~nm}$ was achieved within a period of less than $30 \mathrm{~s}$ after turning on the diode laser device at room temperature. The optical power 


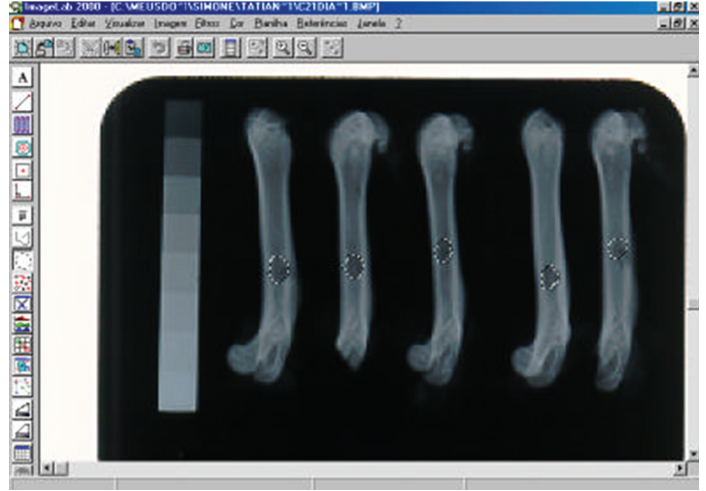

Figure 3

was calibrated using a Newport Multifunction Optical Meter (model 1835C).

Five animals from each group were euthanized at 7 , 14, and 21 days after surgery. The femurs were fixed in formol 10\%. Radiographic images of the specimens were obtained using occlusal film (Kodak Ektaspeed EO-41 P, São José dos Campos, SP, Brazil). The following parameters were standardized for all radiographs: film-focus distance of $40 \mathrm{~cm}$, exposure time of $0.25 \mathrm{~s}, 10 \mathrm{~mA}, 65 \mathrm{kVp}$. The digitalized images were measured using a computer program (ImageLab, Softium Sistema de Informática Ltda).

The average density of the femur was compared with the average density of an aluminum scale to determine the equivalence in millimeters of aluminum. An area of the bone defect related to the area of bone neoformation was bounded (Figure 3). An image pattern was chosen to compare with the $\mathrm{X}$-Ray images. The calibration system eliminates the variations in radiographic processing. The area corresponding to the surgical defect resulted in a value of optical density (OD) that refers to BMD. The protocol was based on the study of Prado-Filho and Sterman [13].

\section{Statistical Analysis}

The densitometric results were compared between groups by analysis of variance (ANOVA) and the Tukey test, with the level of significance set at 5\%, yielding an $F$ value equal to 21.84 , greater than the critical $F(1.99)$, implying a statistically significant difference between groups and time points.

\section{Results}

The absolute values of the BMD analysis allowed the assessment of bone healing by comparing the $\mathrm{C}, \mathrm{Ca}, \mathrm{La}$, and $\mathrm{LaCa}$ groups in relation to the experimental periods (Table 2 ). This analysis showed that bone repair occurred gradually in all groups. At seven days, the LaCa group had the highest BMD values (Table 2). At 14 days, the results were similar in all groups. At 21 days, the values of BMD for the $\mathrm{LaCa}$ group were statistically significant compared to other groups. During this period, the values of La group were lower than observed in $\mathrm{Ca}$ and $\mathrm{LaCa}$ groups (Figure 4).
TABLE 2: Average and standard deviation of BMD (mm/Al).

\begin{tabular}{lccc}
\hline Groups & 07 days & 14 days & 21 days \\
\hline Control $(\mathrm{C})$ & $8 \pm 2$ & $14 \pm 3$ & $18 \pm 5$ \\
Calcitonin $(\mathrm{Ca})$ & $10 \pm 3$ & $16 \pm 1$ & $24 \pm 4$ \\
Laser $(\mathrm{La})$ & $9 \pm 2$ & $15 \pm 2$ & $21 \pm 2$ \\
LLLT and Calcitonin $(\mathrm{LaCa})$ & $14 \pm 3^{*}$ & $15 \pm 2$ & $28 \pm 1^{*}$
\end{tabular}

*Statistically significant values, $P \leq 0,005$.

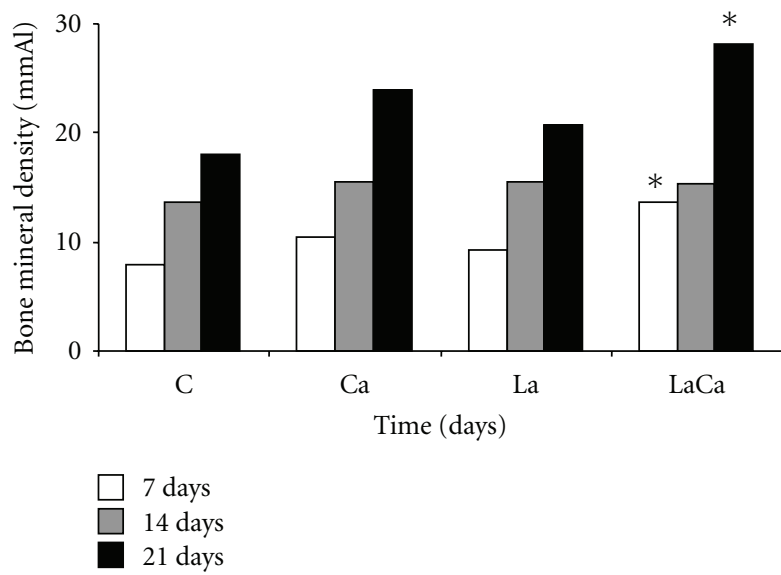

FIGURE 4

BMD gradually increased over time in all groups. However, compared with treated groups, significantly higher values of BMD were obtained for the $\mathrm{LaCa}$ ( 7 and 21 days). The average densitometric values in the control group were consistently lower than in all other groups, indicating a lower amount of mineralized tissue in the bone defect area (Table 2; Figure 4).

\section{Discussion}

In this study, authors evaluated densitometrically the effects of LLLT and calcitonin in rat femurs. Bone repair, regardless of how the injury occurred, results from a series of events that are similar to soft tissue repair. These include inflammation, cell proliferation, and tissue remodeling, the latter being characteristic of bone tissue $[2,3]$.

Experimental studies have shown that the femur is a possible anatomical site for the creation of bone defects because it provides adequate irrigation to supply nutrition to the injured tissue and has mechanical stability [14]. Thus, surgical circumscribed bone defects in the femur are recommended with a standard diameter of $3.7 \mathrm{~mm}$, considered critical size [1-3].

The optical densitometry is an effective technique to evaluate BMD. It is a safe, easily applied method, which determines higher mineral density (BMD) by the increase of radiopacity in the radiographic image $[9,13]$. Studies have reported that $\mathrm{BMD}$ values show results similar to those of histological studies [2].

Nonsurgical laser treatment is an excellent alternative to reduce the period of the healing process, considering that it 
is a noninvasive therapy of simple application [8]. Currently, there are experimental and clinical studies showing favorable results with nonsurgical laser application in bone tissue [3, 14-16]. However, no reports of the association of LLLT and medications such as calcitonin were found in the literature $[3]$.

Calcitonin is a potent inhibitor of osteoclastic bone resorption. It is also capable to stimulate osteoblast proliferation and to induce osteogenic effect in cell studies. Calcitonin has been used in the treatment of many bone disorders such as osteoporosis due to its direct inhibitory effect on osteoclasts and capacity to promote bone formation [2, 17].

A study of ovariectomized rats treated with calcitonin showed a decrease in time of bone healing when compared to the control group, confirmed by assessment of BMD, histomorphometric, and histological analysis [2]. However, the results of this study highlight the possible effect of calcitonin in rats without hormone deficiency, differing from the results of Almeida et al. [1] that also studied male rats.

Calcitonin acts at all stages of bone repair. In the early stages, it probably induces the cell differentiation into osteoblasts of precursor cells present in the granulation tissue. In the late period of bone neoformation, it stimulates osteoblastic activity and inhibits osteoclastic action, through antiresorptive properties. Thus, the newly formed bone tissue has a more compact and organized trabeculae $[1,2,14$, 16].

In addition, several studies have reported that the use of LLLT in the early stages of bone repair decreases the total period of osteoid formation. It aids the organization of clot and promotes granulation tissue formation. LLLT also supplies deficient energy to damaged cells, reducing vascular permeability and inflammatory infiltrate, allowing formation of granulation tissue $[7,10]$.

In order to establish a protocol to compare our results with other studies, an attempt to quantify the amount of energy applied and the irradiation time was made, according to the parameters used by Nascimento et al. [3] and Lopes et al. [8]. All variations of these factors were eliminated, since small doses of energy are not able to stimulate the repair and excessively high doses can inhibit this process [16].

The irradiation protocol used in this study is similar to those used on previous reports $[3,8,14,16]$. Furthermore, the punctual distribution of energy was aimed to irradiate the entire area of the surgical bone defect $[3,12,14,18]$.

The combination of calcitonin and LLLT provided the best results, particularly within 21 days, showing higher rates of BMD than the other experimental groups. A study conducted with male rats with hormonal deficiency observed similar results, when using the combination of LLLT and calcitonin [3]. The results of this study validate the hypothesis that the combination of these therapies is effective in bone repair and allows greater bone formation in reduced time. Although administration of calcitonin is indicated to treat a hormone deficiency, its use in the treatment of experimental bone defects in normal rats showed that this medication contributed to the significant increase in BMD in all periods. Consequently, administration of calcitonin could be recommended in situations where there is need to reduce the time of bone healing and increase of bone density, frequently observed in cases of dental and orthopedic treatments.

It is possible that the effect of LLLT on bone regeneration depends not only on the total dose of irradiation, but also on the time and mode of irradiation $[3,16]$. Many studies have indicated that irradiated bone, mostly with infrared wavelengths, has increased osteoblastic proliferation, collagen deposition, and bone neoformation, when compared to nonirradiated bone $[16,19]$.

\section{Conclusion}

Densitometric analysis of bone experimental defects in rats showed that LLLT $(830 \mathrm{~nm})$ combined with calcitonin improved bone repair and reduced the duration of the bone repair process.

\section{References}

[1] J. D. Almeida, E. A. L. Arisawa, R. F. da Rocha, and Y. R. Carvalho, "Effect of calcitonin on bone regeneration in male rats: a histomorphometric analysis," International Journal of Oral and Maxillofacial Surgery, vol. 36, no. 5, pp. 435-440, 2007.

[2] E. A. L. Arisawa, A. A. H. Brandão, J. D. Almeida, and R. F. da Rocha, "Calcitonin in bone-guided regeneration of mandibles in ovariectomized rats: densitometric, histologic and histomorphometric analysis," International Journal of Oral and Maxillofacial Surgery, vol. 37, no. 1, pp. 47-53, 2008.

[3] S. B. Nascimento, C. A. Cardoso, T. P. Ribeiro et al., "Effect of low-level laser therapy and calcitonin on bone repair in castrated rats: a densitometric study," Photomedicine and Laser Surgery, vol. 28, no. 1, pp. 45-49, 2010.

[4] D. Ofluoglu, E. Karadag-Saygi, C. Canbulat, O. H. Gunduz, E. Kul-Panza, and G. Akyuz, "Early effect of nasal salmon calcitonin on the bone marker Crosslaps," Rheumatology International, vol. 26, no. 4, pp. 288-291, 2006.

[5] M. Bulbul, C. Z. Esenyel, M. Esenyel, S. Ayanoglu, B. Bilgic, and T. Gulmez, "Effects of calcitonin on the biomechanics, histopathology, and radiography of callus formation in rats," Journal of Orthopaedic Science, vol. 13, no. 2, pp. 136-144, 2008.

[6] X. Li, X. Luo, N. Yu, and B. Zeng, "Effects of salmon calcitonin on fracture healing in ovariectomized rats," Saudi Medical Journal, vol. 28, no. 1, pp. 60-64, 2007.

[7] C. B. Lopes, M. T. T. Pacheco, L. Silveira Jr., J. Duarte, M. C. T. Cangussú, and A. L. B. Pinheiro, "The effect of the association of NIR laser therapy BMPs, and guided bone regeneration on tibial fractures treated with wire osteosynthesis: raman spectroscopy study," Journal of Photochemistry and Photobiology B, vol. 89, no. 2-3, pp. 125-130, 2007.

[8] C. B. Lopes, A. L. B. Pinheiro, S. Sathaiah, J. Duarte, and M. C. Martins, "Infrared laser light reduces loading time of dental implants: a raman spectroscopic study," Photomedicine and Laser Surgery, vol. 23, no. 1, pp. 27-31, 2005.

[9] G. Giro, D. Gonçalves, C. E. Sakakura, R. M. R. Pereira, E. Marcantonio Júnior, and S. R. P. Orrico, "Influence of estrogen deficiency and its treatment with alendronate and estrogen on bone density around osseointegrated implants: radiographic study in female rats," Oral Surgery, Oral Medicine, Oral Pathology, Oral Radiology and Endodontology, vol. 105, no. 2, pp. 162-167, 2008. 
[10] R. F. Z. Lizarelli, K. P. C. Ciconelli, C. A. Braga, and R. J. Berro, "Low-powered laser therapy associated to oral implantology," in Lasers in Dentistry V, Proceedings of SPIE, pp. 69-73, San Jose, Calif, USA, January 1999.

[11] Y. Ueda and N. Shimizu, "Pulse irradiation of low-power laser stimulates bone nodule formation," Journal of Oral Science, vol. 43, no. 1, pp. 55-60, 2001.

[12] A. N. Silva Jr., A. L. B. Pinheiro, M. G. Oliveira, R. Weismann, L. M. P. Ramalho, and R. A. Nicolau, "Computerized morphometric assessment of the effect of low-level laser therapy on bone repair: an experimental animal study," Journal of Clinical Laser Medicine and Surgery, vol. 20, no. 2, pp. 83-87, 2002.

[13] J. C. Prado-Filho and F. A. Sterman, "Evaluation of mineral bone density in thoroughbred horses under an initial trainning programme," Brazilian Journal of Veterinary Research Animal Science, vol. 41, no. 6, pp. 384-388, 2004.

[14] A. L. B. Pinheiro, F. D. A. Limeira Jr., M. E. M. Gerbi et al., "Effect of 830-nm laser light on the repair of bone defects grafted with inorganic bovine bone and decalcified cortical osseous membrane," Journal of Clinical Laser Medicine and Surgery, vol. 21, no. 6, pp. 383-388, 2003.

[15] M. E. M. Gerbi, A. L. B. Pinheiro, C. Marzola et al., "Assessment of bone repair associated with the use of organic bovine bone and membrane irradiated at $830 \mathrm{~nm}$," Photomedicine and Laser Surgery, vol. 23, no. 4, pp. 382-388, 2005.

[16] A. L. B. Pinheiro and M. E. M. M. Gerbi, "Photoengineering of bone repair processes," Photomedicine and Laser Surgery, vol. 24, no. 2, pp. 169-178, 2006.

[17] F. A. Claro, J. R. S. Lima, M. A. C. Salgado, and M. F. Gomes, "Porous polyethylene for tissue engineering applications in diabetic rats treated with calcitonin: histomorphometric analysis," International Journal of Oral and Maxillofacial Implants, vol. 20, no. 2, pp. 211-219, 2005.

[18] A. P. Lirani-Galvão, V. Jorgetti, and O. Lopes da Silva, "Comparative study of how low-level laser therapy and low-intensity pulsed ultrasound affect bone repair in rats," Photomedicine and Laser Surgery, vol. 24, no. 6, pp. 735-740, 2006.

[19] D. A. A. P. Oliveira, R. F. de Oliveira, R. A. Zangaro, and C. P. Soares, "Evaluation of low-level laser therapy of osteoblastic cells," Photomedicine and Laser Surgery, vol. 26, no. 4, pp. 401404, 2008. 


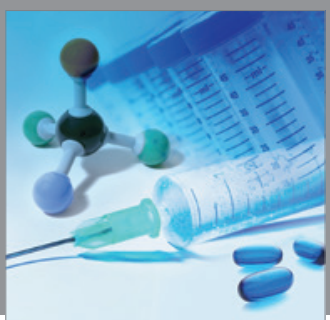

International Journal of

Medicinal Chemistry

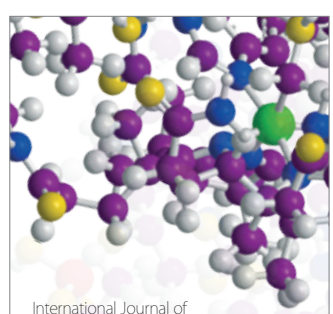

Carbohydrate Chemistry

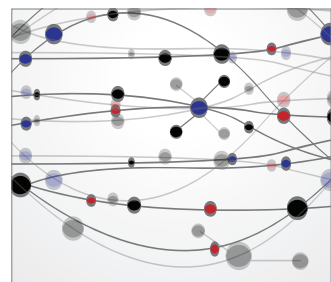

The Scientific World Journal
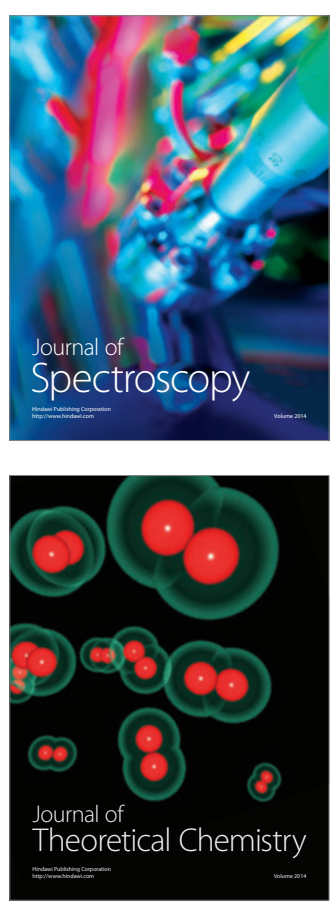
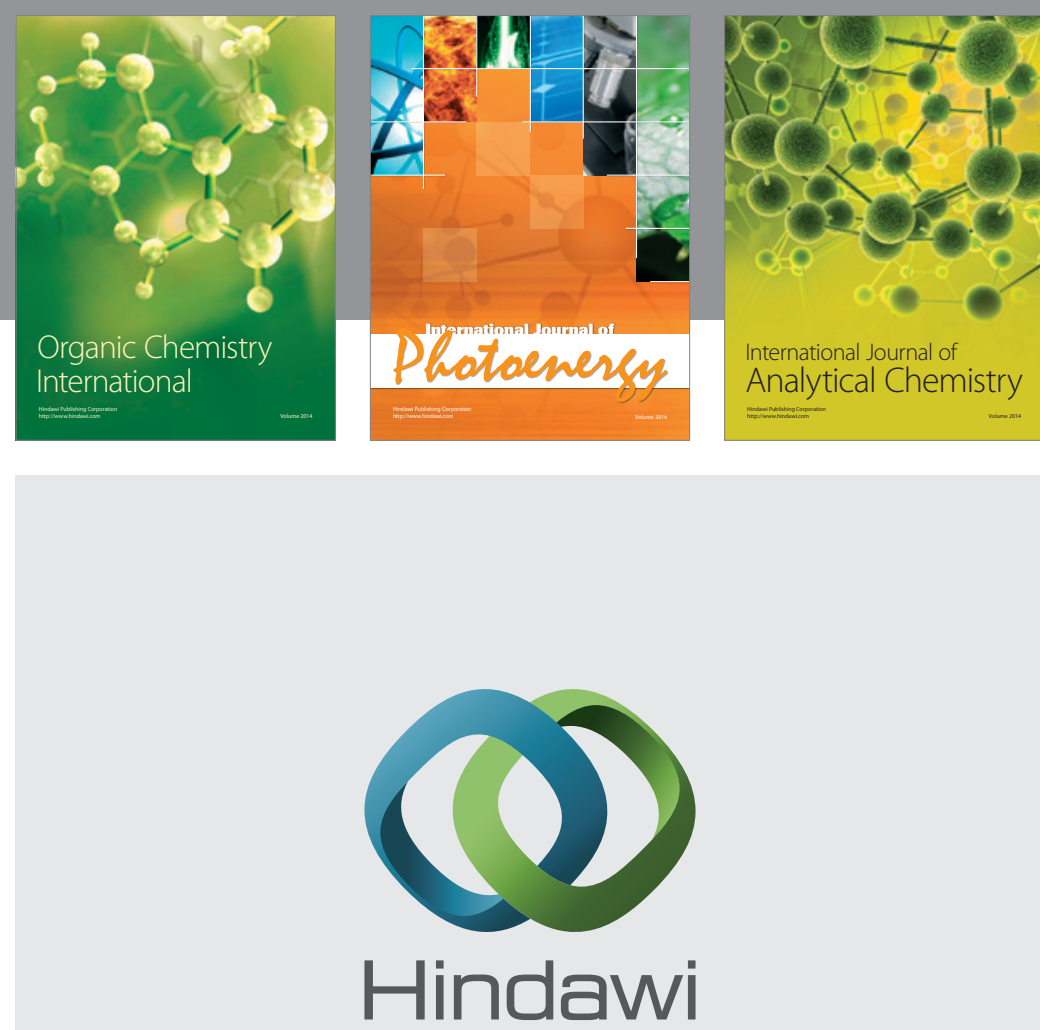

Submit your manuscripts at

http://www.hindawi.com
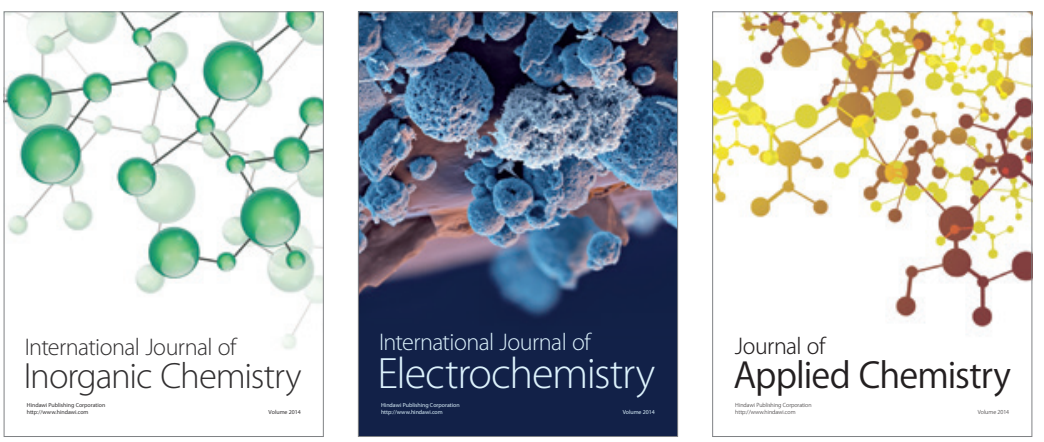

Journal of

Applied Chemistry
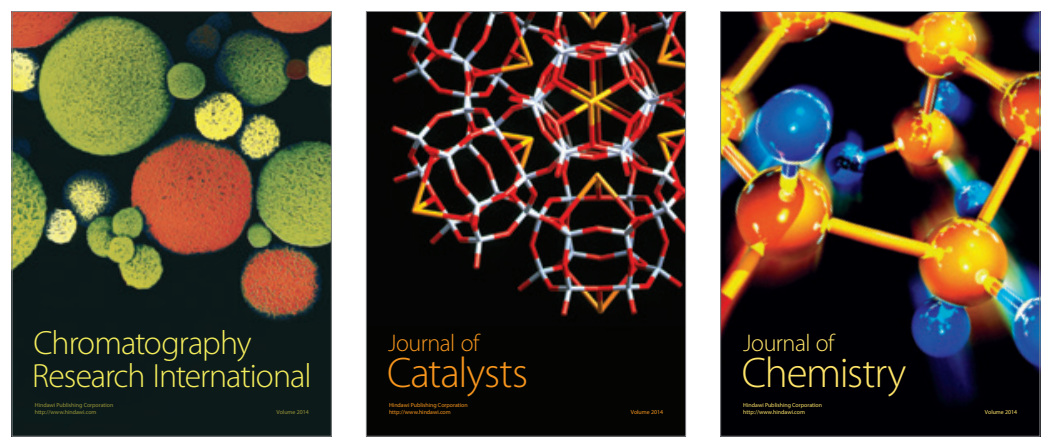
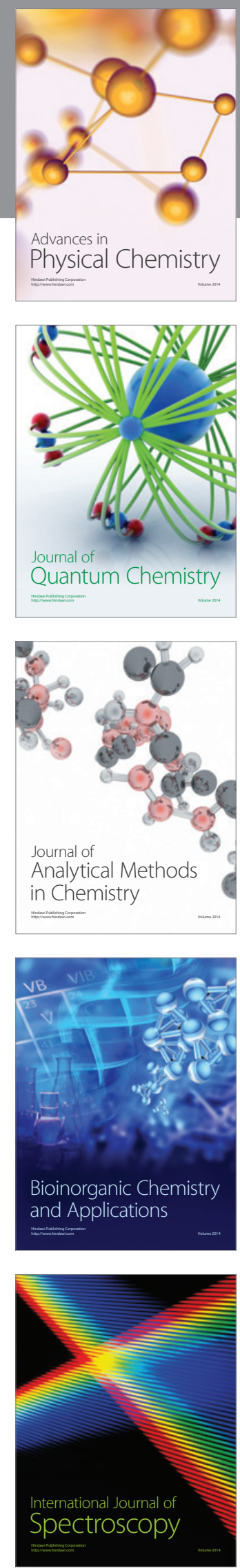